129 SURVIVAL OUTCOMES OF PATIENTS WITH T4/STAGE 4A CERVICAL CANCER: A 10 YEAR REAL WORLD DATA ACROSS 4 NHS TRUSTS

'S Adeleke*, 'A Davis, ${ }^{2} \mathrm{~J}$ Shi, 'S Susanna George, 'H Zahid, 'A Choy, 'K Nathan,

${ }^{1} \mathrm{R}$ Jyothirmayi, ${ }^{1}$ J Summers, ${ }^{1} \mathrm{C}$ Mikropoulos. ${ }^{1}$ Maidstone Hospital, UK; ${ }^{2}$ Guy's Hospital, UK

\subsection{6/ijgc-2021-ESG0.10}

Introduction/Background* There are around 3,200 new cases of cervical cancer(CC) each year in the UK and it accounts for at least $2 \%$ of all new cancer cases. Management can involve surgery for early-stage disease or chemoradiation (CRT). In our regional cancer network, we explored the disease course, changing management paradigm, complications and outcomes of patients with T4/stage IVA(FIGO 2009) CC over a 10 -year period.

Methodology We carried out a retrospective, multicentre analysis between Jan 12010 and Dec 31 2020. The project included 4 NHS trusts in the region. Overall, 274 cervical cancer cases were reviewed, 35 cases were stage 4A and therefore suitable for analysis. In each case, treatment protocol (including radiotherapy dose/fraction), urinary diversion procedure, presence of fistula and survival outcomes were reviewed. Kaplan-Meier analysis and Logrank tests were used to compare survival distributions.

Result(s)* Median PFS of those who received 50.4Gy with a boost(external beam $14.4 \mathrm{~Gy}$ in $8 \#$ to include compromised parametria or $19.5 \mathrm{~Gy}$ in $3 \# \mathrm{HDR}$ ) vs no boost was 89.8 months vs 6 months, $p=0.0462$. Their median OS were 90.6 months vs 8.8 months respectively $p=0.0216$. Those treated with palliative intent had a median OS of 8 months while those with a poor performance status $(\mathrm{PS}>2)$ and hence managed with best supportive care(BSC) died within 6.5 months of presentation. $7 / 35(20 \%)$ patients had evidence of either vesico-vaginal $(\mathrm{n}=5)$ or recto-vesico-vaginal fistula $(n=2) .2$ patients developed fistula as a result of therapy. Presence of fistula (9 vs 12.42 months, $p=0.4374$ ) and hydronephrosis (8.8 vs 13.1 months, $p=0.3668$ ) did not lead to a significant difference in OS. Patient age $(\geq 50 \mathrm{yrs})$ and high PS were found to be associated with increased risk of death, $p<0.0330$ )

Conclusion* Admittedly, there were fewer patients in some of the groups analysed however, the data has shown the impact of presence or absence of boost treatment on OS and PFS. Those treated with palliative intent and BSC unsurprisingly but predictably had poorer outcomes. Presence of fistula or hydronephrosis did not significantly impact patient OS. However, higher age at diagnosis and fitness was associated with increased risk of death.

\section{SURVIVAL OUTCOMES IN CERVICAL CANCER: WHAT FACTORS AFFECT RECURRENCE?}

${ }^{1} \mathrm{C}$ Wang ${ }^{*},{ }^{1 ; 2} \mathrm{~B}$ Lester, ${ }^{3} \mathrm{~L}$ Huang, ${ }^{3} \mathrm{~S}$ Sun, ${ }^{1,4} \mathrm{JJ}$ Ko. ${ }^{1}$ University of British Columbia, Medicine, Vancouver, Canada; ${ }^{2} B C$ Cancer, Radiation Oncology, Abbotsford, Canada; ${ }^{3}$ University of the Fraser Valley, Mathematics and Statistics, Abbotsford, Canada; ${ }^{4} B C$ Cancer, Medical Oncology, Abbotsford, Canada

\subsection{6/ijgc-2021-ESG0.11}

Introduction/Background* The purpose of this study is to determine the disease, and treatment characteristics of stage IB-IV cervical cancer associated with survival differences.

\section{a}

Radical 50.4Gy + Boost vs 50.4Gy no boost
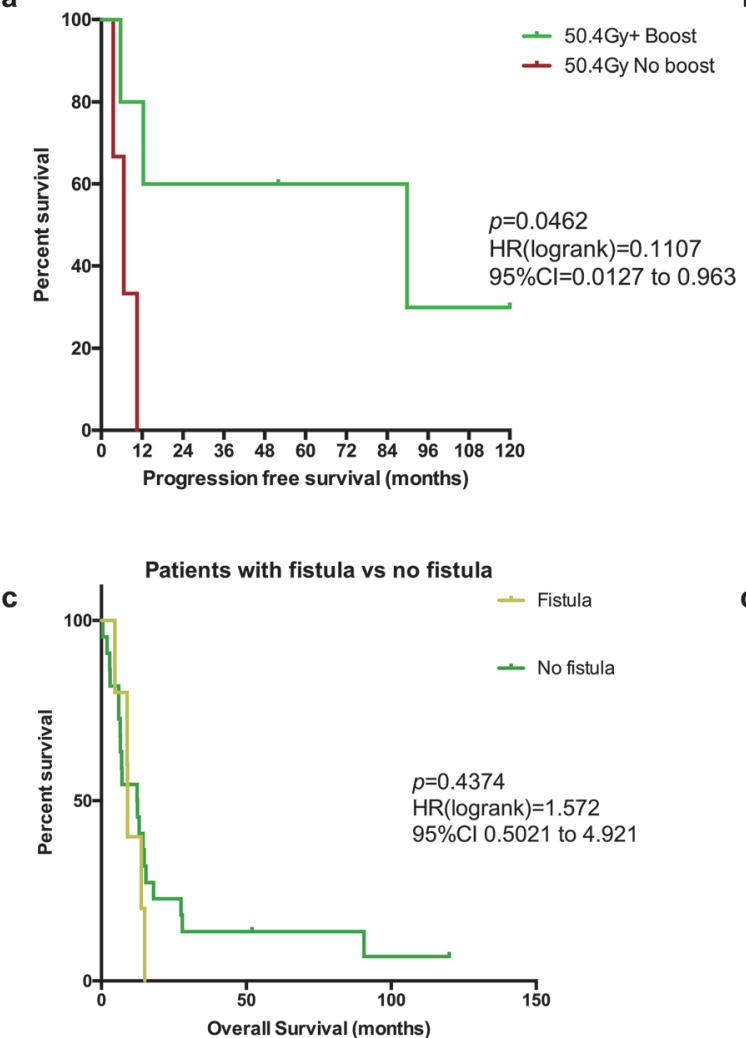

b

Radical 50.4 Gy + Boost vs 50.4 Gy no boost

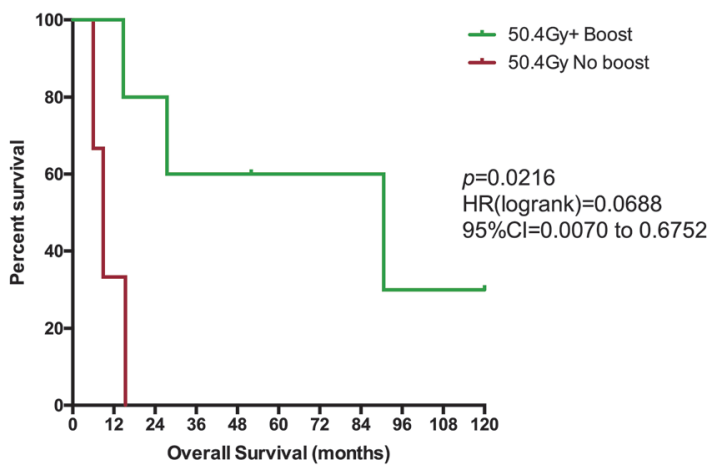

Hydronephrosis vs No hydronephrosis

d

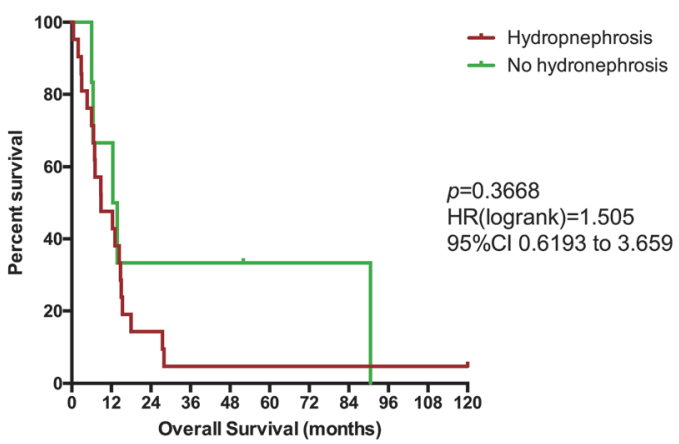

Abstract 129 Figure 1 Progression free and overall survival Kaplan-Meier curves 
Methodology A retrospective chart review on cervical cancer patients in BC between January 1, 2010 and December 31, 2017 was done. Demographic data, treatment details and covariates of prognostic signifikance were collected. Data analysis included logistic regression, multivariate Cox regressions, pairwise comparisons and 2-tailed t tests as appropriate.

Result(s)* 780 patients were examined (stage I 31.5\%, II $20.0 \%$, III $34.5 \%$, IV 3.3\%). Survival outcomes are presented

\section{Abstract 132 Table 1}

\begin{tabular}{|c|c|c|c|c|}
\hline \multicolumn{2}{|c|}{ Characteristic } & \multirow{2}{*}{$\begin{array}{c}n(\%) \\
132(16.9)\end{array}$} & \multirow{2}{*}{$\begin{array}{c}\begin{array}{c}\text { Median Overall } \\
\text { Survival (months) }\end{array} \\
\text { NA }\end{array}$} & \multirow[t]{2}{*}{ P Value } \\
\hline Lymphovascular Invasion & Yes & & & \\
\hline & No & $240(30.8)$ & NA & $<0.0001$ \\
\hline & Unknown & $408(52.3)$ & 95.1 & \\
\hline \multirow[t]{3}{*}{ P16 } & Positive & $208(26.7)$ & NA & \\
\hline & Negative & $21(2.7)$ & 17.2 & $<0.0001$ \\
\hline & Unknown & $551(70.6)$ & NA & \\
\hline \multirow[t]{2}{*}{ Surgical Resection } & Yes & $186(23.8)$ & NA & $<0.0001$ \\
\hline & No & $594(76.2)$ & 124 & \\
\hline \multirow{2}{*}{$\begin{array}{c}\text { Radical } \\
\text { Radiotherapy }\end{array}$} & Yes & $640(82.1)$ & NA & $<0.0001$ \\
\hline & No & $140(17.9)$ & 21.6 & \\
\hline \multirow[t]{3}{*}{ Type of RT } & EBRT + Brachytherapy & $523(67.1)$ & 29.2 & $<0.0001$ \\
\hline & EBRT alone & $194(24.9)$ & NA & \\
\hline & No RT & $63(8.1)$ & --- & \\
\hline \multirow{2}{*}{$\begin{array}{c}\text { Concurrent } \\
\text { Chemotherapy }\end{array}$} & Yes & $227(29.1)$ & NA & $<0.0001$ \\
\hline & No & $553(70.9)$ & 44.6 & \\
\hline \multirow{2}{*}{$\begin{array}{l}\text { Type of Concurrent } \\
\text { Chemotherapy }\end{array}$} & Weekly Cisplatin & $538(69.0)$ & NA & $<0.0001$ \\
\hline & $\begin{array}{c}\text { Other } \\
\text { Not applicable }\end{array}$ & $\begin{array}{c}26(3.3) \\
229(29.3)\end{array}$ & 26.8 & \\
\hline \multirow{2}{*}{$\begin{array}{c}\text { Weeks of } \\
\text { Concurrent Cisplatin }\end{array}$} & 5 weeks & 408 & NA & $<0.0001$ \\
\hline & $<5$ weeks & 143 & NA & \\
\hline \multirow{3}{*}{$\begin{array}{c}\text { Chemotherapy Dose } \\
\text { Reduction }\end{array}$} & Yes & $87(11.2)$ & NA & 0.18 \\
\hline & No & $466(59.7)$ & NA & \\
\hline & Not applicable & $227(29.1)$ & & \\
\hline \multirow{3}{*}{$\begin{array}{c}\text { Peri-RT } \\
\text { Chemotherapy* }\end{array}$} & Yes & $39(5.0)$ & NA & 0.11 \\
\hline & No & $144(18.5)$ & NA & \\
\hline & Not Applicable & $597(76.5)$ & & \\
\hline \multirow{3}{*}{$\begin{array}{l}\text { First Line Systemic } \\
\text { Therapy in recurrence }\end{array}$} & Carboplatin/paclitaxel/bevacizumab & $57(7.3)$ & 40.1 & 0.03 \\
\hline & All other & $80(10.3)$ & 24.8 & \\
\hline & Not applicable & $643(82.4)$ & & \\
\hline
\end{tabular}

\section{Abstract 132 Table 2}

\begin{tabular}{|c|c|c|c|c|c|c|c|c|c|}
\hline \multirow{2}{*}{\multicolumn{2}{|c|}{ Characteristic }} & \multicolumn{8}{|c|}{ Median Overall Survival (Months) } \\
\hline & & Stage I & $P$ value & Stage II & $P$ value & Stage III & $P$ value & Stage IV & $P$ value \\
\hline Lymphovascular Invasion & $\begin{array}{l}\text { Yes } \\
\text { No } \\
\text { Unknown }\end{array}$ & $\begin{array}{l}\text { NA } \\
\text { NA } \\
\text { NA }\end{array}$ & 0.005 & $\begin{array}{l}\text { NA } \\
\text { NA } \\
\text { NA }\end{array}$ & 0.05 & $\begin{array}{l}104 \\
\text { NA } \\
121\end{array}$ & 0.04 & $\begin{array}{l}12.2 \\
23.7 \\
12.0\end{array}$ & 0.21 \\
\hline P16 & $\begin{array}{l}\text { Positive } \\
\text { Negative } \\
\text { Unknown }\end{array}$ & $\begin{array}{l}\text { NA } \\
\text { NA } \\
\text { NA }\end{array}$ & 0.67 & $\begin{array}{l}\text { NA } \\
24.2 \\
\text { NA }\end{array}$ & 0.01 & $\begin{array}{l}71.6 \\
16 \\
124.4\end{array}$ & $<0.001$ & $\begin{array}{l}11.70 \\
8.23 \\
15.36\end{array}$ & 0.19 \\
\hline Surgical Resection & $\begin{array}{l}\text { Yes } \\
\text { No }\end{array}$ & $\begin{array}{l}\text { NA } \\
\text { NA }\end{array}$ & 0.009 & $\begin{array}{l}\text { NA } \\
\text { NA }\end{array}$ & 0.73 & $\begin{array}{l}\text { NA } \\
121\end{array}$ & 0.24 & $\begin{array}{l}28.9 \\
11.4\end{array}$ & 0.067 \\
\hline $\begin{array}{l}\text { Radical } \\
\text { Radiotherapy }\end{array}$ & $\begin{array}{l}\text { Yes } \\
\text { No }\end{array}$ & $\begin{array}{l}\text { NA } \\
\text { NA }\end{array}$ & 0.90 & $\begin{array}{l}\text { NA } \\
16.5\end{array}$ & 0.005 & $\begin{array}{l}124.4 \\
14.3\end{array}$ & $<0.001$ & $\begin{array}{l}43.2 \\
10.0\end{array}$ & $<0.001$ \\
\hline Type of RT & $\begin{array}{l}\text { EBRT + Brachytherapy } \\
\text { EBRT alone }\end{array}$ & $\begin{array}{l}\text { NA } \\
\text { NA }\end{array}$ & 0.60 & $\begin{array}{l}\text { NA } \\
61.5\end{array}$ & $<0.001$ & $\begin{array}{l}\text { NA } \\
22\end{array}$ & $<0.001$ & $\begin{array}{l}28.5 \\
11.5\end{array}$ & 0.02 \\
\hline $\begin{array}{l}\text { Concurrent } \\
\text { Chemotherapy }\end{array}$ & $\begin{array}{l}\text { Yes } \\
\text { No }\end{array}$ & $\begin{array}{l}\text { NA } \\
\text { NA }\end{array}$ & 0.97 & $\begin{array}{l}\text { NA } \\
23.8\end{array}$ & $<0.001$ & $\begin{array}{l}\text { NA } \\
21.2\end{array}$ & $<0.001$ & $\begin{array}{l}25.3 \\
11.2\end{array}$ & $<0.001$ \\
\hline $\begin{array}{l}\text { Type of Concurrent } \\
\text { Chemotherapy }\end{array}$ & $\begin{array}{l}\text { Weekly Cisplatin } \\
\text { Other }\end{array}$ & $\begin{array}{l}\text { NA } \\
81.5\end{array}$ & 0.32 & $\begin{array}{l}\text { NA } \\
\text { NA }\end{array}$ & 0.65 & $\begin{array}{l}\text { NA } \\
39.8\end{array}$ & 0.15 & $\begin{array}{l}21.9 \\
14.5\end{array}$ & 0.19 \\
\hline $\begin{array}{l}\text { Weeks of } \\
\text { Concurrent Cisplatin }\end{array}$ & $\begin{array}{l}5 \text { weeks } \\
<5 \text { weeks }\end{array}$ & $\begin{array}{l}\text { NA } \\
\text { NA }\end{array}$ & 0.16 & $\begin{array}{l}\text { NA } \\
\text { NA }\end{array}$ & 0.003 & $\begin{array}{l}\text { NA } \\
65\end{array}$ & 0.007 & $\begin{array}{l}28.1 \\
15.5\end{array}$ & 0.78 \\
\hline
\end{tabular}


for the cohort as a whole, and stratified by stage of diagnoses in table 1 and 2 respectively. Decreased overall survival was associated with lymphovascular invasion and p16 negativity, however when stratified by stage, LVI significantly impacted survival in stage I to III patients only. Increased survival was associated with surgical resection, radical radiotherapy (RT), brachytherapy, concurrent cisplatin and 5 weeks of chemotherapy (vs. $<5$ weeks). When stratified by stage, surgical resection only improved survial in stage I patients, with no significant difference in any other stage. The use of radical RT, brachytherapy, and concurrent chemotherapy did not show survival differences in stage I disease, but did in stage II to IV. As a whole, peri-RT chemotherapy was not associated with survival benefit in adeno/adenosquamous carcinoma. 180 women recurred (23.1\%) with mostly distant metastases $(42.8 \%)$. There was lower incidence of recurrence after primary surgical resection in those with tumor size $<2 \mathrm{~cm}$ vs. tumors $>2 \mathrm{~cm}(4.1 \%$ vs $24.7 \%, \mathrm{p}=0.0004)$. Though only $37.7 \%$ of recurrence/metastases was treated with first-line carboplatin/paclitaxel/bevacizumab, it was associated with better overall survival compared to other regimens (median OS 40.1 vs. 24.8 months, $\mathrm{p}=0.03$ ).

Conclusion* A significant number of women had recurrence (23.1\%), and LVI and p16 negativity is associated with poor survival. Surgical resection in stage I is associated with improved survival but not in stage II to IV. Use of radical chemoradiation treatment is associated with survival differences in stage II to IV disease, but not stage I. First line carboplatin/paclitaxel/bevacizumab for recurrence shows improves survival but only a small proportion of women received it.

\section{DRUG REPURPOSING AS A SOURCE OF INNOVATIVE THERAPIES IN CERVICAL CANCER}

${ }^{1} \mathrm{R}$ Capistrano*, ${ }^{2} \mathrm{~S}$ Paul, ${ }^{3} \mathrm{~A}$ B Boere, ${ }^{1,4} \mathrm{P}$ Pantziarka, ${ }^{5} \mathrm{~S}$ Chopra, ${ }^{3} \mathrm{R}$ Nout, ${ }^{1} \mathrm{G}$ Bouche. ${ }^{1}$ Anticancer Fund, Brussels, Belgium; ${ }^{2}$ Tata Memorial Hospital, Tata Memorial Centre, Homi Bhabha National Institute, Navi Mumbai, India; ${ }^{3}$ Erasmus MC Cancer Institute, Erasmus University Medical Center, Rotterdam, Netherlands; ${ }^{4}$ The George Pantziarka TP53 Trust, London, UK; ${ }^{5}$ Advanced Centre for Treatment, Research and Education in Cancer (ACTREC), Tata Memorial Centre, Homi Bhabha National Institute, Navi Mumbai, India

\subsection{6/ijgc-2021-ESG0.12}

Introduction/Background* Cervical cancer is the fourth cancer in terms of incidence and mortality in women worldwide. Relative to other cancers, there has been limited progress in the discovery of effective new therapies. Drug repurposing is an alternative development pathway that utilise the properties of drugs approved for other diseases and builds on available safety and pharmacological data to develop the drug as a potential (cervical) cancer drug.

We screened the literature to identify drug repurposing opportunities in cervical cancer to inform future research and trials.

Methodology A literature-based approach was undertaken to identify whether the drugs included in ReDO_DB (database of 317 non-cancer drugs on the market with at least one article reporting a possible effect on any cancer type) or CDcervix_DB (database containing 217 drugs approved for one or more malignancies by a regulatory agency, but excluding drugs currently used in cervical cancer). PubMed was queried for each drug and all abstracts were assessed for relevance and

Abstract 143 Table 1 Five examples of repurposing candidates for cervical cancer

\begin{tabular}{|c|c|c|c|c|}
\hline $\begin{array}{l}\text { Drug } \\
\text { Main approved } \\
\text { indications }\end{array}$ & $\begin{array}{l}\text { Proposed mechanism } \\
\text { of action in cervical } \\
\text { cancer }\end{array}$ & $\begin{array}{l}\text { Potential role } \\
\text { single agent/ } \\
\text { radiosensitizer/ } \\
\text { immunomodulation }\end{array}$ & Proposed setting(s) & $\begin{array}{l}\text { Cervical } \\
\text { cancer } \\
\text { trials }\end{array}$ \\
\hline $\begin{array}{l}\text { Nelfinavir } \\
\text { HIV }\end{array}$ & $\begin{array}{l}\text { PI3K-Akt inhibition and } \\
\text { induction of } \\
\text { endoplasmic reticulum } \\
\text { stress }\end{array}$ & radiosensitizer & with CRT & ongoing \\
\hline $\begin{array}{l}\text { Hydralazine \& } \\
\text { valproate } \\
\text { Hypertension \& } \\
\text { epilepsy, respectively }\end{array}$ & $\begin{array}{l}\text { HDAC and DNA } \\
\text { methyltransferase } \\
\text { inhibition }\end{array}$ & $\begin{array}{l}\text { radiosensitizer } \\
\text { immunomodulation }\end{array}$ & $\begin{array}{l}\text { with CRT, } \\
\text { adjuvant, } \\
\text { recurrent/ metastatic }\end{array}$ & yes \\
\hline $\begin{array}{l}\text { Sonidegib } \\
\text { Basal cell carcinoma }\end{array}$ & $\begin{array}{l}\text { smoothened inhibition } \\
\text { and radiosensitizer }\end{array}$ & radiosensitizer & $\begin{array}{l}\text { with CRT, } \\
\text { adjuvant }\end{array}$ & no \\
\hline $\begin{array}{l}\text { Plerixafor } \\
\text { Mobilisation of } \\
\text { haematopoietic stem } \\
\text { cells }\end{array}$ & $\begin{array}{l}\text { prevention of CRT- } \\
\text { induced CXCL12/CXCR4 } \\
\text { signalling }\end{array}$ & $\begin{array}{l}\text { radiosensitizer } \\
\text { Immunomodulation }\end{array}$ & with CRT & no \\
\hline $\begin{array}{l}\text { Cetuximab } \\
\text { Squamous cell head } \\
\text { and neck cancer }\end{array}$ & $\begin{array}{l}\text { EGFR inhibition and } \\
\text { radiosensitizer }\end{array}$ & radiosensitizer & $\begin{array}{l}\text { with CRT, } \\
\text { recurrent/ metastatic }\end{array}$ & yes \\
\hline
\end{tabular}

